\title{
The STAGE Platform: User Engagement of Older People Through Co-Design and Extended Access to Streamed Cultural Events
}

\author{
Luigi BIOCCA ${ }^{\mathrm{a}, 1}$ and Nicolò PARACIANI ${ }^{\mathrm{a}}$ \\ ${ }^{a}$ National Research Council, Institute of Heritage Science - CNR ISPC
}

\begin{abstract}
This paper illustrates the aim, concept and scope of the STAGE project as a complementary way of exploiting leisure. This is achieved through accessing cultural events for the benefit of older users in addition to the standard direct participation. Older users could not always avail such events within reach, due to several reasons - mobility and family problem, lacking infrastructure and transportation, affordability. Accessing cultural events via streaming is not an alternative or substitute of direct participation, but it is an additional way of attending that extends the audience, the range of selectable events - also internationally - and the participation options through time. Platform development was carried out with a significant and supportive user engagement of older people through the co-design methodology.
\end{abstract}

Keywords. Older users, user engagement, co-design, cultural events, streaming, mobility, extended participation.

\section{Introduction}

The STAGE project (Streaming of Theatre and Arts for old aGe Entertainment) was funded within the AAL (Active and Assisted Living) program and lasted 3-years (2016 - 2019).

STAGE provides accessible cultural content via video streaming technology delivered through a Web-based platform. Contents include several types of cultural events (theatre plays, concerts, opera, etc.) for extending opportunities to older users and let them enjoy culture despite possible limiting conditions.

Older end users have been engaged in the design process, giving their preferences and opinions. They have provided useful information for the successful development and final testing of the platform.

${ }^{1}$ Corresponding Author, CNR ISPC, Area Ricerca RM1, Via Salaria km. 29,300 - 00015 Monterotondo Staz., ROMA - Italy; E-mail: luigi.biocca@cnr.it. 


\section{Rationale}

Leisure time can be a beneficial factor in terms of wellbeing, active life, physical and mental health, especially for older users [1-2]. This is widely recognized as a key factor for regenerating health, fitness, physical training, and cognitive stimulation. Leisure should help them explore new knowledge coming from culture, education, travelling and entertainment. It seems appropriate to keep an active mental attitude.

These assumptions specially involve older users, particularly those frailer and at possible risk of isolation.[3].

Older users generally have more leisure, compared to other age segments. This factor can empower their life independence through self-determination of needs, requirements, expectations and choices.

Although interested in culture, older users might not be always able to physically access cultural events due to any occasional restrictions (affordability, mobility problems, lack of transportation/infrastructure, family/health reasons, etc.) [4].

STAGE facilitates enjoying cultural events at home via a specifically designed Webbased streaming platform, offering contents provided by various cultural entities.

\section{The STAGE project outline}

STAGE was funded within the AAL - Active Assisted Living - Program in Europe and carried out from 2016 to early 2019. AAL focuses on promoting older users' quality of life at home by funding specific market-oriented systems, products, and services. [5].

Co-design is a recommended bottom-up methodology to empower older users' participation and contribution to the final product or service prototype [6]. This policy encourages user engagement and raises awareness among older users as to selfdetermination of needs and expectations in daily life at home.

STAGE is an ICT platform providing older users with facilitated access to cultural events via streaming technology (recorded, scheduled and live-streamed videos).

The platform was implemented through the co-design methodology by involving older users in the definition of design and functional features. This would then gradually proceed to the final implementation from older users' requirements.

Older users were thus recruited for co-design and trials on a voluntary basis. An interest in culture was a fundamental criterion. During the trial, they received training for proper usage of the platform. After this, we collected their feedback on usability and accessibility.

\subsection{Objectives}

The ambition of STAGE is to empower older users' online access to cultural contents theatre plays, concerts, opera performance and museum exhibits. This content is easy, co-designed, customized and affordable. STAGE serves a double purpose: satisfying the unmet need for remote access to culture and stimulating cognitive abilities. Indeed, studies have shown the beneficial effect of entertainment and engagement in cultural activities on older users' mental health [7]. 
Older users appear generally interested in culture and entertainment, and usually have enough free time for that. However, some of them might face obstacles that can severely hinder participation.

The barriers can be health conditions, affordability issues, lack of infrastructure and transportation, mobility limitations and lack of physical accessibility of event venues. All these factors sometimes are very difficult to overcome [8].

Therefore, STAGE provides a cultural offer fitting to older users who could be facing such issues. However, STAGE could also be potentially open to other age groups.

The STAGE ICT platform has the final goal of finalizing its product/service to the marketplace.

The platform is equipped with a customized interface and makes content available for use on common digital devices connected to the Internet.

It also provides information content about available events that enables users to post comments on videos and rate their appreciation and experience.

Thus, STAGE also improves older users' social participation and helps reduce digital divide by increasing their computer literacy.

The STAGE platform was intended to offer affordable digital tickets for single events, prepaid event packages, weekly and monthly packages. Payments can be processed through PayPal; a voucher system was proposed as an additional feature.

\section{Platform architecture}

The platform - being Web-based - is compatible with standard operating systems and connected devices.

It is developed through open-source programming languages and an extended Application Programming Interface (API) that ensures interoperability of the platform, making use of Docker containers to improve deployment and performance - see Fig.1. The platform adopts the Drupal 7 Content Management Framework. [9].

As database system, STAGE uses MySQL and runs according to efficient load balancing techniques.

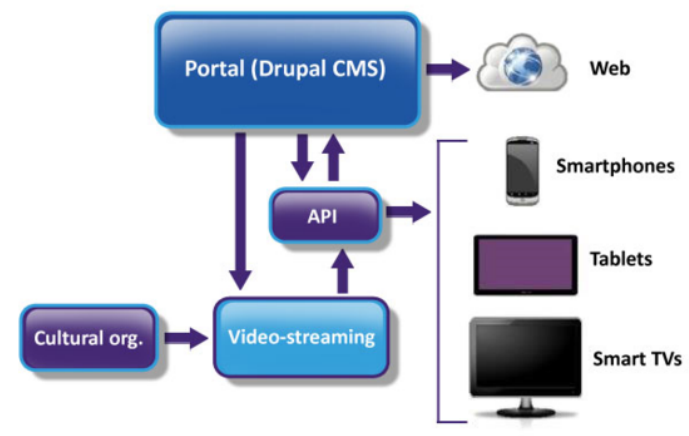

Figure 1. Platform architecture. 


\section{Older user involvement}

Older users' engagement took place in three steps as follows:

- User requirements;

- Co-design;

- Test and final evaluation.

\subsection{User recruitment}

The initial selection criteria were:

- $60+$ years old;

- $\quad$ no serious cognitive diseases;

- coming from different social and cultural contexts in terms of education, computer literacy and family composition;

- gender balanced.

60 persons aged $60+$ participated on a voluntary basis from three project partner countries - Italy, Hungary, and Cyprus - in the overall project evaluation process.

Interest to cultural events was a prerequisite for joining the project trial . A preliminary survey was initially made among potential older participants to investigate through a questionnaire their attitudes, habits, actual knowledge, and level of interest as to different cultural events.

After some introductory project meetings to present objectives, scope, and relevance of the users' role in co-design, we administered a 36-questions questionnaire to older users including the following sections:

- Section 1 (4 questions): residence information;

- Section 2 (6 questions): personal information;

- Section 3 (15 questions): social participation and recreational activities;

- Section 4 (6 questions): preferences related to the STAGE platform;

- Section 5 (5 questions): technical information.

We explored habits, attitudes, behaviors, and expectations of older users as regards to leisure, social and cultural activities: this survey helped defining a general user profile for the project use cases and scenarios. Thus, data on social participation and engagement were relevant to the link with cultural interest.

We also identified specific questions on preferences about the STAGE platform and cultural content .

We highlight some of the most relevant results from the preliminary survey as follows:

- Health: more than $50 \%$ stated to be in good health conditions and nearly $3 \%$ suffered major disabilities; the remaining $46 \%$ reported minor disabilities: about $65 \%$ of them had motor disabilities, $15 \%$ hearing disability, $15 \%$ with visual disability and $6 \%$ with multiple disabilities; 
- Mobility: 84\% reported going out at least once a day;

- $\quad$ Proximity and service access within $500 \mathrm{mt}$. related to: public transportation (bus stops or subway stations); social and daily activities (mall, gardens, community services, social center for older adults etc.);

cultural activities;

- $\quad 94 \%$ had at least one bus stop or subway station and $89 \%$ a social spot within reach; instead, only $27 \%$ had a cultural activity within reach, while the remaining $73 \%$ needed to cover a larger distance to reach one;

- Participation in social and recreational activities: older users reported watching TV, watching movies, reading, walking and surfing the Web as their top five rated leisure activities. Despite a relatively low attendance to cultural events, most older users expressed interest in watching the cultural content STAGE would offer as streamed video events, especially for theatre plays and concerts.

Older users reported the following reasons for low attendance:

$35 \%$ for budget restrictions (e.g. tickets too expensive), $29 \%$ for mobility problems (e.g. help needed when going out), 18\% for personal and/ or health problems (e.g. arthritis) and 18\% for other reasons.

- Usage of tickets reservation services: $30 \%$ of the respondents used ticket reservation services, mostly for cinemas and theatres;

- Feeling of age limitations in cultural participation: 59\% considered aging not a limit to participation in cultural events, while $29 \%$ experienced limitations, although not severe ones;

- Level of interest and participation in cultural activities: 7\% reported an active interest and direct participation; $41 \%$ reported an active interest but could not participate; $18 \%$ expressed an occasional interest and 34\% were not interested at all. Most older users reported theatre and opera as best preferences of cultural events;

- Potential interest in using STAGE to watch various cultural events via streaming: $85 \%$ of users reported to be potentially interested in such an application with the following motivations:

- improving knowledge and keeping up with technology (45\%);

- opportunity to watch cultural events at home (16\%);

- keeping an active brain (10\%);

- entertainment and socialization (10\%);

- economic benefits (4\%).

Moreover, $49 \%$ perceived that engaging in an online cultural community would positively improve social relationships. More than $59 \%$ were interested in learning resources for culture and more than $63 \%$ would like to access educational information about STAGE cultural events.

- Most rated expectations from STAGE: $57 \%$ of older users reported all three proposed options - "easy to use", "price" and "broad offer" as "very important" or "important";

- Computer literacy: familiarity and unfamiliarity with social networks and Web were both reported by $39 \%$ of users; the remaining $22 \%$ did not know; 
- Devices use and frequency online: computer 52\%, smartphone $27 \%$, tablet $12 \%$ and laptop $9 \%$; $77 \%$ had an Internet connection.

- Among those with Internet connection, $41 \%$ could use it autonomously, 31\% only partially and $28 \%$ could not, while $54 \%$ is regularly connected (at least once a day), $39 \%$ never and $7 \%$ only rarely.

- Overall confidentiality with devices: all respondents were confident/very confident with TV, $42 \%$ with PC/laptop, $29 \%$ with smartphone and $21 \%$ with tablet.

From the preliminary user feedback and evaluation we reported a general picture of older users potentially interested in experiencing STAGE for the delivery of cultural content at home via streaming. This would help them acquire more knowledge, be more confident with media and learn new opportunities for being more proactive in daily life.

This seemed to suggest that STAGE could be an effective supplement to direct participation for bringing worldwide cultural events at home, thus providing an easier media to keep older adults' cultural interest alive, while at the same time overcoming obstacles and limitations.

\subsection{Co-design}

User requirements were defined for implementing the STAGE prototype.

Focus was on a draft Graphical User Interface (GUI) as result of older users' feedback. The GUI is the core of the platform visual look and was presented to users as a set of mock-ups through static pictures (a mock-up is a model of a design or a device, in this case an image containing the design of the platform.).

We developed and administered a dedicated questionnaire to users to collect their preferences.

This consisted of questions on the suitability of the mock-ups content as to clarity and intelligibility of design elements.

The results of this analysis were a prerequisite for the development of a new GUI design.

By understanding and testing the mock-ups, older users can provide valuable feedback about functionality, usability, and the design idea.

The advantages of using mockups were considerable, particularly as follows:

- mock-ups encourage criticism from older users because mock-ups are low-cost and easily modifiable;

- $\quad$ mock-ups give rise to an effective discussion and feedback between designer and older user because of fast understanding and intuitiveness. For example, a printed mock-up and a pen enable the designer and older user to put and compare ideas directly on paper.

Moreover, mock-ups can be shown immediately to a variety of audiences (i.e. older users from different countries)

The mock-up process shows how the system should look like for the best usability. 
Once the mock-ups were presented to older users for feedback, the designers worked through continuous adjustments until the final implementation step.

Figures 2 and 3 show mock-ups for the advanced search page and for the Web video player, respectively.

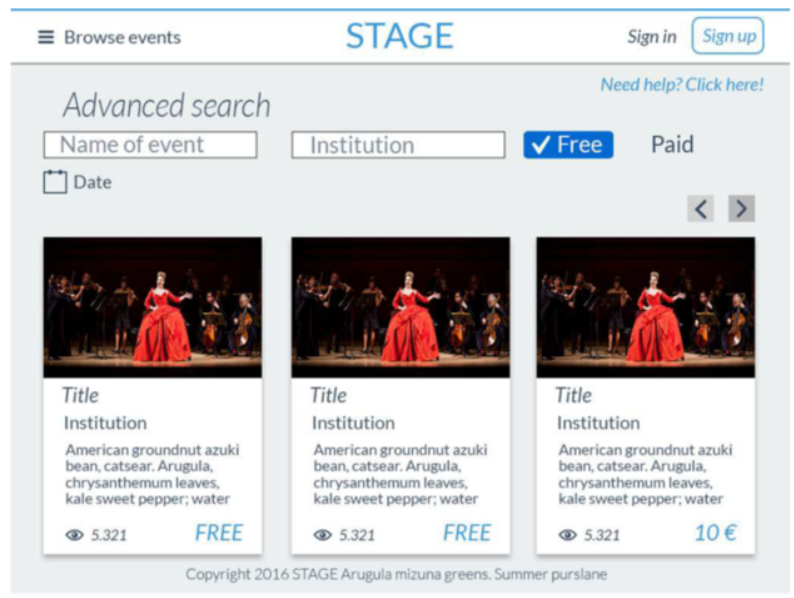

Figure 2. The advanced search page.

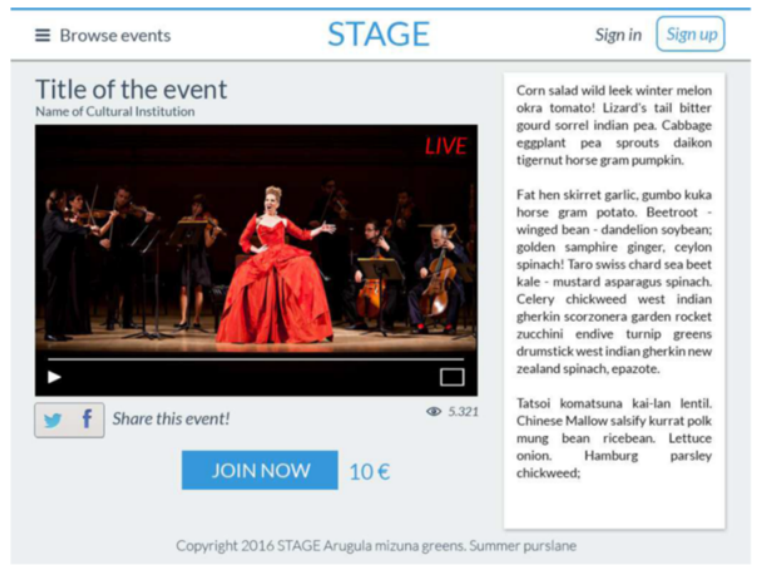

Figure 3. The Web Player page.

We administered a specific questionnaire to older users about the GUI mock-ups to explore the suitability of the graphical interface. This included a set of specific questions of accessibility, for suggesting improvements and changes.

Questions about accessibility and intelligibility were based on the Web Content Accessibility Guidelines (WCAG) [10].

We mainly considered the homepage clarity, the suitability of color contrast, and font sizes and the intelligibility of written texts.

We asked the following set of questions on each applicable mock-up page: 

a. Is the purpose of the page and of its elements clear at first impact?
b. Is the text understandable?
c. Is the font size comfortable to read?
d. Is the combination of colors appropriate?
e. Is the size of buttons / arrows / icons appropriate?

We also included questions on specific user preferences such as appropriateness and suitability of menu types and easy-to-understand interactive elements.

The questionnaire consisted of 73 questions and 16 pictures of mock-ups.

Without going deeper into the details of users' feedback, we illustrate a significant example of the home page evolution: After the users' feedback on the first version, text was added to give users a short description of STAGE.

However, the final version displays a shortened text, which is more effective and intuitive - see Fig. 4.
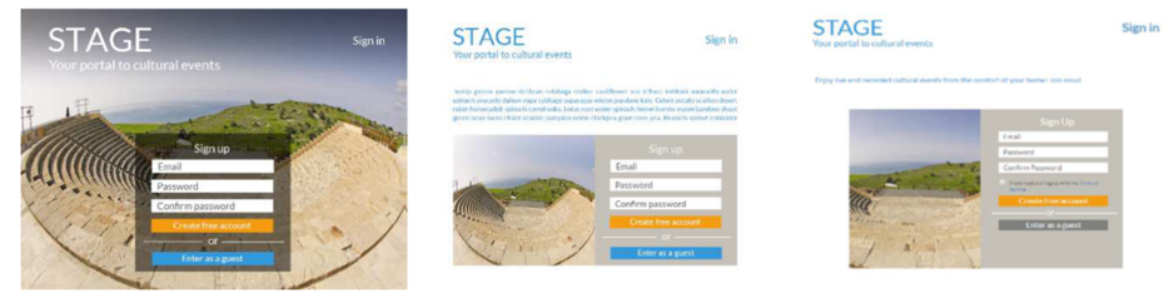

Figure 4. Home page evolution: first, intermediate and final version from the left.

Older users requested to amend other issues: the 'search by category' option was added ( $10 \%$ of the interviewees asked for it); the event preview box was added with information about the access mode of videos on demand ("Free" or "Paid"); the user menu items were reformulated and a history of watched videos was added.

\subsection{Test and final evaluation}

The final trial evaluation took place after delivery of appropriate training and assistance to allow older users to easily explore the platform. This meant that older users were getting gradually confident with the platform from the beginning.

Thus, the final evaluation of the platform is the result of the previous phases of user engagement. This was carried out on extensive training sessions based on the prerequisites and conditions established in the evaluation framework.

Older users tested usability of the platform, together with preferred types and periodic updates of the cultural events. This would be a key for future positive platform acceptance.

We have conducted trials and tests in three different European countries, with their different cultural contexts.

We have performed iterative testing and evaluations of the prototype and targeted system concepts, device usability, and user interaction. 
The iterative testing led to optimization of the prototype for the field evaluation. We conducted the overall pilot trial for 1 year.

59 users participated in the third and final trial phase; 10 of them were recruited as first-time testers with no training.

During the testing period, we collected questionnaire data from three feedback sessions for further platform development and upgrade.

\subsubsection{Summary of final evaluation}

Users reported a positive appreciation of the platform functionality, use and targets during the overall final evaluation of the third trial. During feedbacks, discussions and co-design sessions, we detected a growing interest in the overall product and service STAGE can deliver, especially in terms of additional content and exploitation of live streaming.

Older users expect a larger selection of content; thus, a larger network of cultural event providers is essential to extend the offer and ensure various content with better quality.

We can summarize some recommendations from the final evaluation users' feedback as follows:

- $\quad$ Improving the search feature to facilitate content search;

- Customizing payment system through PayPal according to the visual features of the "virtual store";

- Improving standard PayPal interface with larger font sizes, while ensuring local language translations are available;

- Implementing alternative voucher payment and/or gift cards;

- Optimizing platform's architecture for a faster content downloading and a better user experience, considering any limited broadband connection coverage in some areas;

- Upgrading "Social” features (e.g. dedicated social network, forums, chat rooms etc.) to empower users to interact with each other and exchange thoughts and experiences.

- $\quad$ Upgrading platform accessibility to relevant WCAG guidelines.

\section{Conclusions}

We can conclude that most older users quite appreciated STAGE's scope and purpose during the trial.

The platform appears designed with a user-centric vision that emphasizes older users' co-design rather than a 'ready-to-use' top-down approach.

Importantly, older users were made aware of their role and the potential of this service seems very promising. Indeed, it aims to extend older users' proactivity in leisure time by supplementing - but not excluding - social and direct participation in different cultural events worldwide. 


\section{References}

[1] Ragheb, M.G., Griffith, C.A.: The contribution of leisure participation and leisure satisfaction to life satisfaction of older persons. J. Leisure Res. 14(4), 295 (1982)

[2] Toepoel, V.: Cultural participation of older adults: investigating the contribution of lowbrow and highbrow activities to social integration and satisfaction with life. Int. J. Disabil. Hum. Dev. 10(2), 123129 (2011)

[3] Toepoel, V.: Ageing, leisure, and social connectedness: how could leisure help reduce social isolation of older people? Soc. Ind. Res. 113(1), 355-372 (2013)

[4] http://www.comres.co.uk/polls/arts-council-england-older-people-poll/. Accessed August 2020

[5] Filizola, N., The solutions and findings of the AAL Programme. February 2020 (available at: https://www.eesc.europa.eu/sites/default/files/files/presentation_filizola_24-02-2020.pdf)

[6] AA.VV, AAL Market and Investment Report technopolis group, May 2018

[7] SAlA, G., JOPP, D., GOBET, F., OGAWA, M., ISHIOKA, Y., MASUI, Y., INAGAKI, H., NAKAGAWA, T., YASUMOTO, S., ISHIZAKI, T., ARAI, Y., IKEBE, K., KAMIDE, K., GONDO, Y. The Impact of Leisure Activities on Older Adults' Cognitive Function, Physical Function, and Mental Health, doi: 10.1371/journal.pone.0225006. eCollection 2019.PMID: 31703115 (2019)

[8] BIOCCA, L., PARACIANI, N., PICENNI, F., PADUlA, M., CARUSO, G., CHIARIGLIONE, L., KOWALSKA, A., FLOREA, M., KAPOURANIS, I., The STAGE Project: Tailored Cultural Entertainment for Older Adults via Streaming Technology, Human Aspects of IT for the Aged Population. Applications, Services and Contexts. ITAP (2017). Lecture Notes in Computer Science, vol 10298. Springer, Cham. https://doi.org/10.1007/978-3-319-58536-9_39. (2017)

[9] Patel, S. K., Rathod, V. R., Prajapati, J. B.: Performance Analysis of Content Management SystemsJoomla, Drupal and WordPress. Int. J. Comp. Appl. 21(4), 39-43 (2011)

[10] https://www.w3.org/TR/WCAG20/. Accessed August 2020 